\title{
12 year old with dizziness, anemia and jaundice
}

\author{
Tess Woehrlen $^{1 *}$, Helene Tigchelaar ${ }^{2}$ and Nirupama Kannikeswaran ${ }^{2}$ \\ ${ }^{1}$ Pediatric Resident, Division of Pediatric Education, Children's Hospital of Michigan, USA \\ ${ }^{2}$ Pediatrics and Emergency Medicine, Carman and Ann Adam Department of Pediatrics, Division of Emergency Medicine, Children's Hospital of Michigan, USA
}

\section{Presentation}

A 12-year-old African American adolescent girl presents to the Emergency Department with the chief complaint of dizziness. She has a 6 week history of vague symptoms including decreased appetite, dizziness, fatigue and weakness. Mom noticed yellowing of the eyes and pallor of the palms of the hands for the last two weeks. Today she had a syncopal episode that lasted a few minutes without any seizure like activity. Her past medical history is significant for intermittent asthma, eczema, seasonal allergies and cataract of the left eye. Family history is significant for anemia, rheumatoid arthritis, Crohn's disease, fibromyalgia, pulmonary embolism, heart disease and hypertension.

Vitals on presentation to the ER are as follows: BP: $110 / 68 \mathrm{mmHg}$, HR $100 \mathrm{bpm}$, RR $22 \mathrm{brpm}$, temp $37.1^{\circ} \mathrm{C}$, Ht $157 \mathrm{~cm}$, Wt $38 \mathrm{~kg}$, BMI 15.4 $\mathrm{kg} / \mathrm{m}^{2}$ (16 $\%$ ile). Physical examination is significant for scleral icterus with pallor of the conjunctiva and nail beds and mild tachycardia. There is no lymphadenopathy, hepatosplenomegaly, petechiae, bruising or ecchymosis noted. And complete neurological exam did not reveal any focal deficits.

Diagnostic studies obtained show a hemoglobin of $6.8 \mathrm{gm} /$ $\mathrm{dL}$, hematocrit of $20.3 \%$, MCV $106.3 \mathrm{FL}$, RDW 22.9\%, reticulocyte count of $1.3 \%$, a white blood cell count (WBC) of $3.8 \mathrm{~K} / \mathrm{CUMM}$, an absolute neutrophil count of $1824 \mathrm{~K} / \mathrm{CUMM}$ and a normal platelet count. Serum lactate dehydrogenase is elevated to 2,057 units/L. Liver enzymes and serum uric acid are within normal limits. The total serum bilirubin is $4.1 \mathrm{mg} / \mathrm{dL}$ with direct fraction of $0.2 \mathrm{mg} / \mathrm{dL}$. An abdominal ultrasonography and chest radiograph are normal.

\section{Discussion}

To further evaluate the macrocytic anemia, serum and red blood cell folate and B12 level were obtained. B12 level was low at $142 \mathrm{pg} /$ $\mathrm{mL}$ (reference range 211-911) while folate levels were within normal limits. Review of the blood smear showed red blood cell morphology with significant anisocytosis, macro-ovalocyts and tear drop cells. Occasional schistocytes, rare basket cells and nucleated red blood cells were also noted. WBC morphology was overall normal, with rare hypersegmented neutrophils. Platelets were normal in number and morphology with the exception of a few large platelets. The low vitamin B12 level and the findings on the blood smear are consistent with vitamin B12 deficiency.

In order to determine the etiology, homocysteine and methylmalonic acid levels were ordered. Urine methylmalonic acid was elevated at 15,100 micromole/L (reference range 1.6-29.7). Serum parietal cell antibody test was positive and intrinsic factor blocking antibody was elevated at $173.1 \mathrm{AU} / \mathrm{mL}$ (reference range: 0.0-0.1 AU/ $\mathrm{mL}$ ), diagnostic of pernicious anemia.

Pernicious Anemia (PA) is an autoimmune disorder in which destruction of the gastric mucosa and the parietal cells leads to hypochlorhydria as well as impaired secretion of intrinsic factor, which is essential for vitamin B12 uptake within the terminal ileum $[1,2]$. There appears to be a genetic susceptibility to pernicious anemia with an increased frequency in familial inheritance and an up to $30 \%$ increased association with other autoimmune neuroendocrinopathies such Hashimoto's Thyroiditis, Type 1 Diabetes Mellitus, Addison Adrenal Insufficiency, Celiac Disease, Sjogern Syndrome and vitiligo $[3,4]$. The inheritance pattern, however, remains unknown [5].

Pernicious anemia primarily affects the neurologic and hematological systems. The initial symptoms such as the weakness seen in our patient, decreased concentration, headache and chest pain or palpitations are usually secondary to the anemia and are non-specific.

Vitamin B12 deficiency may affect both the posterior and lateral columns of the spinal cord, leading to subacute combined degeneration of the spinal cord which presents clinically as impaired vibration, proprioception and sensory changes in the distal extremities. Lesions may also be present in the cerebrum, causing developmental delay, personality change and psychiatric symptoms such as depression $[3,5]$. These nervous systems changes, if left untreated, can progress to axonal death. At this late stage, treatment with Vitamin B12 injections may not completely reverse the nervous system damage [5].

\section{Diagnosis}

The diagnosis of vitamin B12 deficiency requires a low serum B12 level $(<148 \mathrm{ng} / \mathrm{l})$ in conjunction with an elevated serum homocysteine or methylmalonic acid level [4]. To diagnose PA, autoantibodies to parietal cells and atrophic gastritis must be present [5]. The preferred laboratory test is anti-intrinsic factor antibody which is greater than $95 \%$ specific and if positive, a diagnosis of pernicious anemia is assured [3]. An increase in fasting gastrin levels and a decrease in pepsinogen 1 levels are seromarkers that can also be used to help make the diagnosis. Atrophic gastritis is a histological diagnosis made based on the findings of mucosal atrophy with sparing of the gastric antrum, enterochromoffin-like cell hyperplasia and hypochlorhydria on biopsy [5].

\section{Treatment}

PA is treated with $1000 \mathrm{mCg}$ of cyanocobalamin administered either intramuscularly or subcutaneously, every week for 1 month and then monthly thereafter. A complete blood count should be repeated

${ }^{\star}$ Correspondence to: Tess Woehrlen, Pediatric Resident, Division of Pediatric Education, Children's Hospital of Michigan, 3901 Beaubien Blvd, Detroit, MI 48201 USA, E-mail: twoehrle@dmc.org

Received: August 20, 2018; Accepted: September 11, 2018; Published: September 14,2018 
10-14 days after starting cyanocobalamin injections. An increase in hemoglobin and a decrease in mean corpuscular volume is a sign of treatment response. Full hematological recovery is anticipated to occur in 8 weeks [4].

\section{Complications}

Long term complications of pernicious anemia include iron deficiency and atrophic gastritis, a complication of the hypochlorhydria secondary to destruction of the patrietal cells, as well as gastric tumors, a complication of the resulting hypergastrinemia. Yearly monitoring with a complete blood count, serum ferritin and cobalamin levels is necessary to monitor replacement treatment and for the onset of iron deficiency [5]. Those with PA are at a $4-7 \%$ higher risk for gastric tumors including carcinoid tumors, adenocarcinoma and nonHodgkin lymphoma. Yearly screening for new onset gastrointestinal (GI) symptoms such as dyspepsia, dysphasia or epigastric pain is indicated. Onset of GI symptoms warrants an immediate endoscopic evaluation [5].

\section{Patient Course}

While admitted to the in-patient unit, the patient was given a vitamin $\mathrm{B} 121000 \mathrm{mCg}$ intramuscular injection with improvement in clinical symptoms and an expected increase in hemoglobin and reticulocyte count to $7.3 \mathrm{~g} / \mathrm{dL}$ and $24.2 \%$ respectively seven days after the injection. Given the family history of autoimmune conditions, pediatric endocrinology and neurology were consulted to further evaluate for coexisting neuroendocrinopathies, the evaluation of which was negative. She was discharged home on hospital day eight with directions to receive Vitamin B12 $1000 \mathrm{mCg}$ injections weekly for 1 month and then monthly thereafter. Since discharge, her hemoglobin and MCV have normalized with complete resolution of symptoms.

\section{Lessons for the Clinician}

- Pernicious anemia is a rare autoimmune disorder in children resulting from malabsorption of vitamin B12.

- The most common symptoms are those associated with the anemia and neurological changes including alteration in vibration and proprioception as well as sensory changes in the lower extremities.

- Diagnosis of pernicious anemia includes macrocytic anemia, low Vitamin B12 level with elevated serum homocysteine or methylmalonic acid level in addition to addition to elevated parietal cell antibodies and atropic gastritis.

- Treatment consists of intramuscular Vitamin B12 injections weekly, followed by monthly.

- Yearly surveillance is mandatory for early detection and treatment of long term complications such as iron deficiency anemia, atropic gastritis and gastric cancers.

\section{References}

1. Andres E, Serraj K (2012) Optimal management of pernicious anemia. Journal of Blood Medicine 3: 97-103.

2. Reynolds E (2006) Vitamin B12, folic acid, and the nervous system. The Lancet Neurology 11: 949-960.

3. Kumar NN (2017) Continuum (Minneapolis, Minn.): Nutrients and Neurology American Academy of Neurology: 822.

4. Dobrozsi S, Flood VH, Panepinto J, Scott JP, Brandow A (2014) Vitamin B12 deficiency: The great masquerader. Pediatr Blood Cancer 61: 753-755.

5. Lahner E, Annibale B (2009) Pernicious anemia: New insights from a gastroenterological point of view. World J Gastroenterol 15: 5121-5128.

Copyright: $₫ 2018$ Woehrlen T. This is an open-access article distributed under the terms of the Creative Commons Attribution License, which permits unrestricted use, distribution, and reproduction in any medium, provided the original author and source are credited. 